\title{
Exploring gender differences in pre-operative emergency room use in an inpatient pacemaker insertion population
}

\author{
Debosree Roy* James Studnicki \\ The Department of Health Services Research, University of North Carolina, Charlotte, United States
}

Received: December 14, 2015

Accepted: February 4, 2016

Online Published: March 14, 2016

DOI: $10.5430 /$ jha.v5n3p56

URL: http://dx.doi.org/10.5430/jha.v5n3p56

\begin{abstract}
Objective: The study is a prospective analysis of the Florida healthcare utilization project database. Records from the state inpatient database (SID) for the year 2010 and the state emergency department database (SEDD) for the years 2009 and 2010 have been used.

Methods: There are 1,796 patients undergoing permanent pacemaker implants in the 2010 Florida inpatient discharge database according to our set inclusion criterion of all discharges with a primary procedure for initial pacemaker implant with diagnosis for bradycardia, heart block or both, in any position, in the SID. Three outcomes (emergency room [ER] history, true emergency and scheduled) were created based on the population's ER experience within an observation period of 365 days. Descriptive statistics were used to describe patient demographics and clinical characteristics in the outcome groups. Binomial logistic regressions have been used to predict risk for inpatient pacemaker in females and our 3 outcome groups. The models have also been replicated using recursive partitioning methods.

Results: Forty six percent of patients receiving a primary pacemaker in our data were women. Three hundred and five patients are scheduled, of which almost $41 \%$ are women; 697 patients are true emergencies, of which almost $45.5 \%$ are women and 769 patients have ER history, of which almost $48 \%$ are women. We found that sex does not affect outcomes. However, patient characteristics other than sex do affect outcomes, e.g., patients with Medicare as their primary payer are almost $65 \%$ less likely to have the ER history when compared with those having private insurance (OR: 0.35; 95\% CI: 0.16-0.74) and likelihood for women to receive pacemakers increases by $64 \%$ in patients having 2 comorbidities on their discharge record when compared with those who have 3 or more comorbidities (OR: 1.64 ; $95 \%$ CI: 1.05-2.58).

Conclusions: Consistent with previous literature, we did not find any significant differences among the sexes for primary pacemaker implants as well as ER use previous to implant. However subtle differences were observed in discrete patient characteristics like comorbidities, race and primary expected payer in sex-based and ER-utilization based groups. Cardiac events display high gender disparity and have high association with ER use. We have not found any previous study exploring these interactions. Future investigations in this subject should involve a larger sample size in order to carry out non-linear models of exploration along with stochastic analyses in order to increase validity of findings.
\end{abstract}

Key Words: Gender differences, Sex differences, Emergency room use, Pacemaker, True emergencies, Scheduled patients, Patient history of emergency room visits

${ }^{*}$ Correspondence: Debosree Roy; Email: deshrag1983@ gmail.com; Address: The Department of Health Services Research, University of North Carolina, Charlotte, United States. 


\section{INTRODUCTION}

Implantation of cardiac pacemaker is the treatment of choice for severe and/or symptomatic bradycardia, heart block or a combination of both. ${ }^{[1]}$ There has been a notable rise in use of implantable pacemakers, a cardiac rhythm management device, after its clinical success in treating bradycardia and conduction disorders of the heart has been evidenced in multiple population based studies and small cohort studies. ${ }^{[2]}$ Additionally, experience from performance and outcomes of this procedure, as well as the monitoring capacity of the device itself, have added to the existent knowledge base about indications for electrophysiological procedures to treat pace and conduction disorders of the heart. ${ }^{[3]}$

A 1988 population survey pegged US prevalence of implantable pacemaker at 2.6 per thousand populations. Prevalence was higher in older adults. Age adjusted estimates from the same survey (Medical Device Supplement to the National Health Interview Survey) was 1.5 times higher in females than males. ${ }^{[4]}$ In the US Medicare population, prevalence of inpatient primary pacemaker, between 1990 and 2000 increased from 325.4 to 504.4 per 100,000 beneficiaries. ${ }^{[5]}$ However, the prevalence of the procedure in men was greater than that in women among Medicare beneficiaries in the same period. ${ }^{[5]}$ From 1993 to 2006, 2.4 million US adults received primary pacemaker in the US, as estimated from national hospital inpatient discharge summaries. ${ }^{[2]}$

A 2002 consensus on guidelines for primary pacemaker reached through collaboration between the American College of Cardiology, the American Heart Association and the North American Society for Pacing and Electrophysiology, had recommendations on appropriate clinical indications for primary pacemaker insertions. The experts participating in this consensus development felt that adults with third degree and advanced second degree atrioventricular (AV) block with $\mathrm{AV}$ block induced bradycardia and symptoms for heart failure, may require permanent pacing. They also felt that adults with chronic bi-fascular and tri-fascular block as demonstrated by asymptomatic first-degree AV block, asymptomatic type -I second degree AV block at the supra-His level and alternating bundle-branch block may require permanent pacing. ${ }^{[6]}$ The recommendations were elaborated upon in a 2008 report on consensus development on indications for permanent pacemaker. The new recommendations for permanent pacemaker included indications for sinus node dysfunction with documented symptomatic bradycardia, which may be induced by prolonged drug therapy for chronic illnesses. ${ }^{[7]}$ It is well understood that changes in AV conduction in open aortic valve replacement also needs to be addressed with permanent pacemaker implants. ${ }^{[8]}$ Other studies have indicated that permanent pacemakers are also used to treat vasovagal (neu-

Published by Sciedu Press trally mediated) syncope,${ }^{[9]}$ and sick sinus syndrome. ${ }^{[10,11]}$ It is interesting to note that most conditions which require cardiac pacing with permanent pacemakers are manifested in symptoms like syncope, pre-syncope, dyspnea and fatigue, ${ }^{[10]}$ which drives emergency room (ER) use in patients with cardiac problems. ${ }^{[12]}$

Symptoms as pre-cursors or manifestations of cardiovascular diseases are often appraised in the ER. ${ }^{[13]}$ ER referrals for hospital admissions are made following determination of a life-threatening condition. ${ }^{[11]}$ Almost $50 \%$ of all non-obstetric admissions in the US in 2006 recorded previous ER visits. ${ }^{[14]}$ Although plenty of studies have made ER utilization their focus to study its impact on costs, ${ }^{[15]}$ few look at the shift in the ER's role in the US healthcare system. ${ }^{[16]}$ Almost $25 \%$ of all acute care outpatient visits in the US take place in the ER. ${ }^{[17]}$ This is because, the ER is more accessible to patients, especially suffering from acute events like those related to cardiac diseases. The ER services are available 24/7, irrespective of insurance status, and as a setting, the ER guarantees the best possible treatment for acute events, given its proximity to the inpatient hospital setting and allied resources. Thus the ER can be perceived of as a safety net to the underserved in need, including women.

Men's and women's health care experiences differ as they age. ${ }^{[18]}$ Sex is a fundamental dimension in agency use, yet it is seldom considered while planning for service provision. ${ }^{[19]}$ These differences lead to disparate health and treatment outcomes between the sexes, especially in the area of cardiac health, which has significant disease burden in the US. Studies have shown differences in symptoms and presenting conditions for cardiac problems between the sexes, which may explain the disparities in outcomes to a certain extent. This observation has motivated population studies to include more women in their cohorts than what was done historically. ${ }^{[20]}$ Women experience symptoms that "deviate" from the ones found in a clinician's guidebook. ${ }^{[21]}$ There are significant differences among men and women in terms of presenting conditions for cardiac events and diseases. Women are less likely to complain of pressure, heaviness, or tightness in the center or left chest when compared to men. They are more likely to complain of mid-back pain and palpitations when compared to men. ${ }^{[22]}$ A cohort study using hospital discharge data found that women were slightly older, experienced recurrent chest pains and had greater symptom duration as characteristics associated with presenting complaints for cardiac events, when compared with men. ${ }^{[23]}$ Reported anatomic, physiologic, biologic and psychosocial sex differences are valid justifications for sex-based parsing out of presenting conditions and diagnosis of cardiac diseases and events. 
The differences in symptoms may lead to differences in optimal healthcare service provision in the field between women and men, which in turn create disparities in disease outcomes. Women are less likely to have an electrocardiogram performed within 10 minutes of hospitalization and are less commonly cared by a cardiologist during their inpatient stay for acute coronary syndromes. ${ }^{[21]}$ A cohort study found that additional diagnostic procedures were performed more frequently on men than women although the initial test positivity results were similar and on follow up, women had a higher rate of myocardial infarction when compared with men. ${ }^{[24,25]}$ Implications for these subtle differences may lie in differences in health services utilization between the sexes. It is well established that women seek medical care more than men. ${ }^{[25]}$ Women seek more physician services, experience more episodes of acute illnesses and need more long-term care over their life span when compared to men. ${ }^{[26]}$ However, with age, the risk for chronic conditions, especially cardiac conditions, increase. As stated earlier, women are less likely than men to receive diagnostic or therapeutic procedures for heart related events than men, although they have a higher risk for mortality from cardiac events and cardiovascular diseases when compared to men. ${ }^{[24]}$

Although there is adequate literature looking at sex differences in cardiac diseases and rhythm management in isolated care settings, there is a dearth of studies looking into the phenomenon from the perspective of a care-continuum. In recent years, cardiac pacing with the use of cardiac pacemakers has increased. ${ }^{[27]}$ The popularity of this procedure makes it ideal for exploring sex differences in cardiac diseases in the continuum of care. The ER is increasingly becoming the gateway to care in the US. Symptoms which manifest conditions with pacing needs, like shortness of breath, syncope, chest pain etc., often prompt people to seek care in the ER. In fact these symptoms may qualify as precedents for ER visits if presented at a doctor's office or an urgent care clinic.

This study aims at looking at sex differences in patient characteristics associated with primary permanent pacemaker insertions in a large hospital discharge database for a particular year. Additionally, the study also looks at ER utilization of this population within a year prior to surgery, compare patients who had prior ER use with those who did not, stratified by sex and predict ER utilization and pacemaker in females as outcomes.

\section{MethodS}

\subsection{Study design}

The study is a prospective database analysis done using deidentified patient records from the Florida healthcare utilization project (HCUP) data. It has been approved by the Insti- tutional Review Board at the university where the research was conducted. Records from the state inpatient database (SID) for the year 2010 and the state emergency department database (SEDD) for the years 2009 and 2010 have been used. The data is event level. There are 31 diagnosis and procedure categories attached with each inpatient discharge record and there are 10 diagnosis and procedure categories attached with each ER discharge record. They are presented as separate and continuous variables. The importance of a particular diagnosis or procedure is determined by its relative position in the hierarchy of diagnoses or procedures. The diagnosis variables are 5-character ICD09 (International Classification of Diseases, version 09) codes for diseases and procedure variables are 4-character ICD09 codes for procedures.

\subsection{Population studied}

All discharges with a primary procedure (first listed procedure) for initial pacemaker implant with any diagnosis (1 through 31 diagnosis) for bradycardia and/or heart block in the SID have been included in the study. This has been done to secure a homogenous population for contrast and comparison analysis between the genders.

\subsection{Study protocol and measures}

Descriptive statistics (frequencies, percentages, means and standard deviations) have been used to look at demographic (age, race, payer), clinical (diagnosis of bradycardia, heart block or both), and utilization (admission type, length of stay, ER utilization) differences associated with primary initial pacemaker and 1 year pre-operative ER use between the genders. Average number of ER visits and average number of days between last ER visit and day of implant, along with corresponding standard deviations have been calculated for patients who have evidence of one or more ER visits at least one day prior to implant.

For the purpose of predictive analysis, the patients have been characterized into three groups depending on their attributes (scenarios) around ER visit (see Figure 1).

These include the group of patients who have visited the ER at least 1 day prior to implant, henceforth known as patients with ER history; patients who received implant on the day of ER visit, henceforth known as true emergencies; and patients who did not have any ER visits prior to implant, henceforth known as scheduled patients.

\subsection{Data analysis}

Risk of a female patient getting an implant has been modeled across the three scenarios of ER visit using binomial logit regressions. Binary variable gender has been treated as an 
approximation for implant events. The effect of categorical race, continuous age, categorical number of ER visits, categorical days in between last ER and implant and categorical comorbidity count has been predicted on probabilities for a female pacemaker among patients characterized as having ER history. The effect of categorical race, continuous age and categorical comorbidity count has been predicted on probabilities for a female pacemaker among patients characterized as true emergencies and patients characterized as scheduled, separately. A binomial logit regression has also been used to predict the effects of ER visit attribute (ER history, true emergencies and scheduled surgery/no ER) on the probability of a female pacemaker, controlling for categorical race, continuous age and categorical comorbidity count. The stochastic method has been supplemented with the use of recursive partitioning in order to devise an algorithmic approach to find significant predictors of the outcomes explored with binomial linear models. However, only the most significant predictors were retained to train the predictive models. We did not draw out decision trees as per norm; rather we have presented the predicted pathways of outcome realization in a tabular form with goodness of split estimates guiding the inclusionary decision. A predictive probability of more than $50 \%$ has been set for inclusionary decision in favor of a partition.

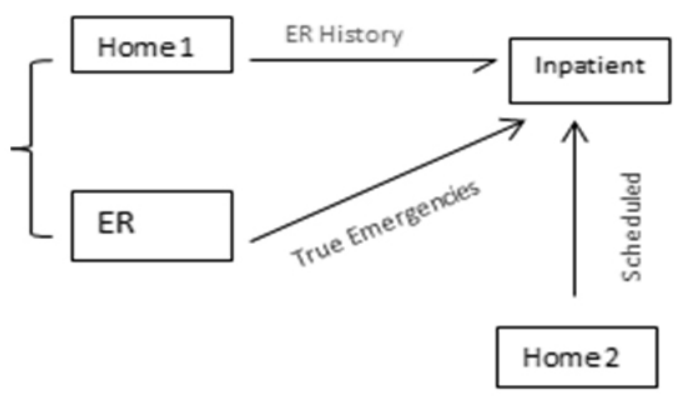

Figure 1. Patient characterization

\section{Results}

Out of the 1,796 patients receiving primary pacemaker in our data, $46 \%$ were women. According to our pre-determined discrete ER utilization-based categorization, 305 patients were scheduled, of which almost $41 \%$ were women; 697 patients were true emergencies, of which almost $45.5 \%$ were women and 769 patients had ER history, of which almost $48 \%$ were women as well (see Figure 2).

Of all patients undergoing primary pacemaker insertion in the inpatient database, $46 \%$ were women. Mean age of women were slightly higher than that of men (78.77 years $v s$. 77.16 years), but the difference was statistically significant $(p=.0014)$. Men tended to have more diagnoses for heart block and bradycardia and heart block than women $(44.59 \%$ Published by Sciedu Press vs. $35.94 \%$ for heart block; $10.69 \%$ vs. $9.52 \%$ for bradycardia and heart block together). Patients were predominantly white and Medicare beneficiaries with $35.69 \%$ of white patients and $40.20 \%$ of Medicare beneficiaries being women. There was a preponderance of emergent admission in our data. This is a discrete variable existent in the SID, which we used in our categorization of patients around their emergency department use; hence, not be confused with the instrumental variables created (true emergencies, ER history and scheduled). Women were observed to have higher counts for all admission type categories than men, i.e., $36.18 \%$ of women had an emergency admission whereas $32.55 \%$ of men had an emergency admission. The difference between the genders in terms of length of stay was unequivocal but statistically significant (4.97 days and 4.41 days in average length of stay for women and respectively, with $p=.0046$ ). A slight difference was observed in terms of ER utilization prior to surgery/ having ER history $(20.72 \%$ for women and $22.70 \%$ for men) and implants on first ER visit day/ true emergencies (17.90\% for women and $21.46 \%$ for men). Most patients had 3 or more comorbidities with $42.32 \%$ women having 3 or more comorbidities on their discharge record and $46.88 \%$ of men having the same on their discharge record. All differences observed between women and men across all categories of comorbidity counts (no comorbidity, 1 comorbidity, 2 comorbidities and 3 and more comorbidities) were statistically significant $(p=.0036)$. When looking at specific comorbidities, women and men showed statistically significant variation in experiencing congestive heart failure (11.64\% vs. $9.80 \%, p=.0002)$, perivascular disease $(4.23 \%$ vs. $7.80 \%$, $p=.0007)$, unspecified hypertension $(27.34 \%$ vs. $28.29 \%$, $p=.0026)$, hypothyroidism (11.14\% vs. 5.85\%, $p \leq .0001)$, renal failure $(8.24 \%$ vs. $12.36 \%, p=.0095)$ and fluid and electrolyte disorders $(11.53 \%$ vs. $8.57 \%, p \leq .0001)$ (see Table 1).

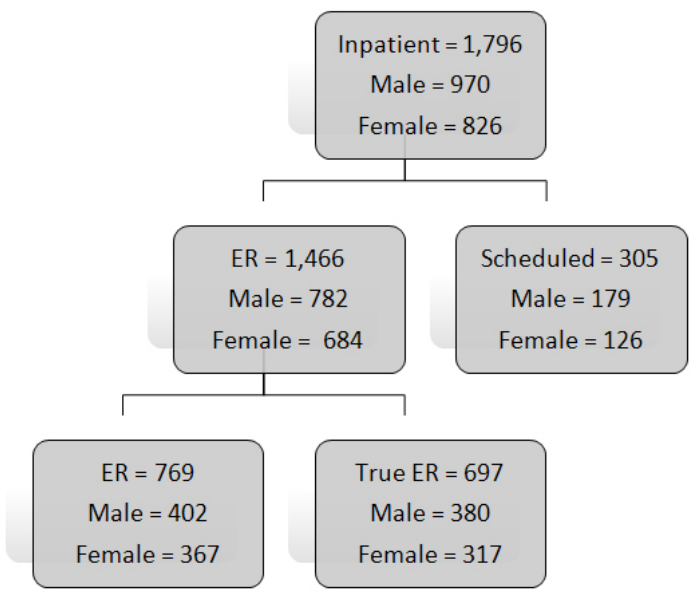

Figure 2. Attrition 
Table 1. Description of patients with primary initial pacemaker insertion with bradycardia or heart block or both by gender

\begin{tabular}{|c|c|c|c|}
\hline Characteristics & Female & Male & $p^{*}$ \\
\hline Sex, n (\%) & $826(45.99)$ & $970(54.01)$ & - \\
\hline Age, mean $(S D)$ & $78.77(10.83)$ & $77.16(10.44)$ & .0014 \\
\hline Bradycardia, n (\%) & $140(9.77)$ & $139(9.70)$ & .0948 \\
\hline Heart Block, n (\%) & $515(35.94)$ & $639(44.59)$ & .0948 \\
\hline Bradycardia and heart block, n (\%) & $171(9.52)$ & $192(10.69)$ & .6328 \\
\hline \multicolumn{4}{|l|}{ Race } \\
\hline White, n (\%) & $637(35.69)$ & $809(45.32)$ & \multirow{3}{*}{.0196} \\
\hline African American, n (\%) & $85(4.76)$ & $72(4.03)$ & \\
\hline Hispanic, n (\%) & $87(4.87)$ & $72(4.03)$ & \\
\hline \multicolumn{4}{|l|}{ Payer } \\
\hline Medicare, n (\%) & $722(40.20)$ & $827(46.05)$ & \multirow{3}{*}{.0123} \\
\hline Medicaid, n (\%) & $29(1.61)$ & $21(1.17)$ & \\
\hline Private Insurance, n (\%) & $61(3.40)$ & $86(4.79)$ & \\
\hline \multicolumn{4}{|l|}{ Admission Type } \\
\hline Emergency, $n(\%)$ & $583(32.55)$ & $648(36.18)$ & \multirow{3}{*}{.1482} \\
\hline Urgent, n (\%) & $153(8.54)$ & $193(10.78)$ & \\
\hline Elective, n (\%) & $87(4.86)$ & $127(7.09)$ & \\
\hline LOS, mean $(S D)$ & $4.97(4.34)$ & $4.41(4.1)$ & .0046 \\
\hline \multicolumn{4}{|l|}{ Comorbidity count } \\
\hline No comorbidity & $10(0.56)$ & $14(0.78)$ & \multirow{4}{*}{.0036} \\
\hline 1 comorbidity & $23(1.28)$ & $41(2.28)$ & \\
\hline 2 comorbidities & $33(1.84)$ & $73(4.06)$ & \\
\hline 3 or more comorbidities & $760(42.32)$ & $842(46.88)$ & \\
\hline ER History, n (\%) & $367(20.72)$ & $402(22.70)$ & .1414 \\
\hline True Emergencies, n (\%) & $317(17.90)$ & $380(21.46)$ & .8616 \\
\hline Scheduled, n (\%) & $126(7.11)$ & $179(7.11)$ & .0882 \\
\hline \multicolumn{4}{|l|}{ Comorbidities, n (\%) } \\
\hline Congestive Heart Failure & $209(11.64)$ & $176(9.80)$ & .0002 \\
\hline Pulmonary circulation disorder & $64(3.56)$ & $48(2.67)$ & .0145 \\
\hline Peri- vascular disorder & $76(4.23)$ & $140(7.80)$ & .0007 \\
\hline Hypertension unspecified & $491(27.34)$ & $508(28.29)$ & .0026 \\
\hline Hypertension complicated & $164(9.13)$ & $231(12.86)$ & .0435 \\
\hline Paralysis & $3(0.17)$ & $5(0.28)$ & .7330 \\
\hline Other neurological disorders & $51(2.84)$ & $62(3.45)$ & .8500 \\
\hline Chronic pulmonary disease & $191(10.63)$ & $213(11.86)$ & .5557 \\
\hline Diabetes uncomplicated & $202(11.25)$ & $264(14.70)$ & .1833 \\
\hline Diabetes complicated & $35(1.95)$ & $57(3.17)$ & .1163 \\
\hline Hypothyroidism & $200(11.14)$ & $105(5.85)$ & $<.0001$ \\
\hline Renal failure & $148(8.24)$ & $222(12.36)$ & .0095 \\
\hline Liver disease & $21(1.17)$ & $13(0.72)$ & .0624 \\
\hline Peptic ulcer w/o bleed & $6(0.33)$ & $8(0.45)$ & .8133 \\
\hline HIV/AIDS & 0 & $2(0.11)$ & .5029 \\
\hline Metastatic cancer & $1(0.06)$ & $4(0.22)$ & .3823 \\
\hline Tumor w/o metastasis & $6(0.33)$ & $18(1.00)$ & .0378 \\
\hline Rheumatoid arthritis & $33(1.84)$ & $21(1.17)$ & .0236 \\
\hline Coagulopathy & $23(1.28)$ & $48(2.67)$ & .0190 \\
\hline Obesity & $64(3.56)$ & $77(4.29)$ & .8814 \\
\hline Weight loss & $17(0.95)$ & $15(0.84)$ & .4139 \\
\hline Fluid and electrolyte disorder & $207(11.53)$ & $154(8.57)$ & $<.0001$ \\
\hline Blood loss anemia & $8(0.45)$ & $4(0.22)$ & .1493 \\
\hline Deficiency anemia & $27(1.50)$ & $20(1.11)$ & .1103 \\
\hline Alcohol abuse & $7(0.39)$ & $22(1.22)$ & .0173 \\
\hline Drug abuse & $5(0.28)$ & $5(0.28)$ & 1.0000 \\
\hline Psychoses & $11(0.61)$ & $6(0.33)$ & .1198 \\
\hline Depression & $93(5.18)$ & $76(4.23)$ & .0132 \\
\hline
\end{tabular}

Note. ${ }^{*}$ Probability of estimates from tests of association $\left(\chi^{2}\right)$ or variance $\left(\mathrm{T}^{2}\right)$ between characteristics and sex 
Table 2. Description of scheduled, true emergencies and patients with ER use history with primary initial pacemaker insertion with bradycardia or heart block or both by gender

\begin{tabular}{|c|c|c|c|c|c|c|c|c|c|}
\hline \multirow{2}{*}{ Characteristics } & \multicolumn{3}{|c|}{ Scheduled } & \multicolumn{3}{|c|}{ True Emergencies } & \multicolumn{3}{|c|}{ ER History } \\
\hline & Female & Male & $p^{*}$ & Female & Male & $p^{*}$ & Female & Male & $p^{*}$ \\
\hline Sex, n (\%) & $126(7.11)$ & $179(10.11)$ & .08 & $317(17.90)$ & $380(21.46)$ & .86 & $367(20.72)$ & $402(22.70)$ & .14 \\
\hline Age, mean $(S D)$ & 77 (11.43) & $76(10.47)$ & .44 & $79(10.04)$ & $77(10.03)$ & .01 & $79(11.44)$ & $76(10.28)$ & .08 \\
\hline \multicolumn{10}{|l|}{ Conditions, n (\%) } \\
\hline Bradycardia & $23(9.24)$ & 25 (10.04) & .22 & $38(7.01)$ & $40(7.38)$ & .45 & $74(11.90)$ & 74 (11.90) & .56 \\
\hline Heart Block & $77(30.92)$ & $124(49.80)$ & .22 & $205(37.82)$ & 259 (47.79) & .45 & $224(36.01)$ & $250(40.19)$ & .56 \\
\hline \multicolumn{10}{|l|}{ Race, n (\%) } \\
\hline White & $111(37.50)$ & $157(53.04)$ & 85 & $234(34.11)$ & $314(45.77)$ & & $280(37.04)$ & 331 (43.78) & \\
\hline African American & $5(1.69)$ & $6(2.03)$ & .85 & $41(5.98)$ & $34(4.96)$ & .13 & $38(5.03$ & $31(4.10)$ & .13 \\
\hline Hispanic & $6(2.03)$ & $11(6.32)$ & & $37(11.15)$ & $26(3.79)$ & & $42(5.56)$ & $34(4.50)$ & \\
\hline \multicolumn{10}{|l|}{ Payer, n (\%) } \\
\hline Medicare & $112(37.97)$ & $151(51.19)$ & & 276 (40.59) & $324(47.65)$ & & $318(42.63)$ & $343(45.98)$ & \\
\hline Medicaid & $3(3.05)$ & $2(1.02)$ & .32 & $7(1.03)$ & $8(1.18)$ & .90 & 19 (2.55) & $11(1.47)$ & .24 \\
\hline Private Insurance & $8(2.71)$ & $19(2.71)$ & & $28(4.12)$ & $37(5.44)$ & & 25 (3.35) & $30(4.02)$ & \\
\hline LOS, mean (SD) & $4.04(5.26)$ & $3.27(3.83)$ & .21 & $4.98(3.92)$ & $4.32(3.05)$ & .01 & $5.34(4.36)$ & $4.97(4.94)$ & .28 \\
\hline \multicolumn{10}{|l|}{ Comorbidity count, n (\%) } \\
\hline No comorbidity & $3(0.98)$ & $2(0.66)$ & & $3(0.43)$ & $3(0.43)$ & & $4(0.52)$ & $9(1.17)$ & \\
\hline 1 comorbidity & $4(1.31)$ & $3(0.98)$ & .04 & $9(1.29)$ & $13(1.87)$ & .30 & $10(1.30)$ & $23(2.99)$ & .07 \\
\hline 2 comorbidities & $6(1.97)$ & $24(7.87)$ & & $13(1.87)$ & $28(4.02)$ & & $14(1.82)$ & $21(2.73)$ & \\
\hline$>3$ comorbidities & $113(37.05)$ & $150(49.18)$ & & $292(41.89)$ & $336(48.21)$ & & $339(44.08)$ & 349 (45.38) & \\
\hline \multicolumn{10}{|l|}{ Comorbidities, n (\%) } \\
\hline Congestive Heart Failure & $22(7.21)$ & $29(9.51)$ & .77 & $80(11.48)$ & $73(10.47)$ & .05 & 104 (13.52) & 73 (9.49) & .0008 \\
\hline Cardiac Arrhythmias & $40(13.11)$ & $62(20.33)$ & .60 & $86(12.34)$ & $113(16.21)$ & .45 & 149 (19.38) & $156(20.29)$ & .61 \\
\hline Valvular disease & $32(10.49)$ & 40 (13.11) & .54 & $51(7.32)$ & $67(9.61)$ & .58 & $75(9.75)$ & $64(8.32)$ & .10 \\
\hline Pul. Circ. disorder & $10(3.28)$ & $14(4.59)$ & .97 & $18(2.58)$ & $12(1.72)$ & .10 & $32(4.16)$ & $21(2.73)$ & .56 \\
\hline Peri-vascular disorder & $11(3.61)$ & $31(10.16)$ & .03 & $26(3.73)$ & $53(7.60)$ & .02 & $36(4.68)$ & $55(7.15)$ & .10 \\
\hline Hypertension unspecified & $77(25.25)$ & $105(34.43)$ & .67 & $196(28.12)$ & 202 (28.98) & .02 & $209(27.18)$ & $196(25.49)$ & .02 \\
\hline Hypertension complicated & $20(6.56)$ & $35(11.48)$ & .41 & $64(9.18)$ & $89(12.77)$ & .30 & $77(10.01)$ & $106(13.78)$ & .08 \\
\hline Paralysis & 0 & 0 & - & $2(0.29)$ & $4(0.57)$ & .69 & $1(0.13)$ & $1(0.13)$ & 1.00 \\
\hline Other neurological disorders & 0 & $9(2.95)$ & .13 & $21(3.01)$ & $20(2.87)$ & .45 & $26(3.38)$ & $33(4.29)$ & .56 \\
\hline Chronic pulmonary disease & $2(0.66)$ & $36(11.80)$ & .82 & $64(9.18)$ & $76(10.90)$ & .95 & $98(12.74)$ & $100(13.00)$ & .56 \\
\hline Diabetes uncomplicated & $24(7.87)$ & $51(16.72)$ & .08 & $81(11.62)$ & 101 (14.49) & .76 & $91(11.83)$ & $108(14.04)$ & .51 \\
\hline Diabetes complicated & $25(8.20)$ & $8(2.62)$ & .76 & $15(2.15)$ & $20(2.87)$ & .76 & $16(2.08)$ & $29(3.77)$ & .09 \\
\hline Peptic ulcer w/o bleed & $1(0.33)$ & 0 & .41 & $3(0.43)$ & $4(0.57)$ & 1.00 & $2(0.26)$ & $4(0.52)$ & .48 \\
\hline HIV/AIDS & $1(0.33)$ & 0 & - & 0 & $1(0.14)$ & 1.00 & 0 & $1(0.13)$ & 1.00 \\
\hline Metastatic cancer & 0 & $1(0.33)$ & 1.00 & 0 & $3(0.43)$ & .25 & 0 & 0 & - \\
\hline Tumor w/o metastasis & $1(0.33)$ & $3(0.98)$ & .64 & $4(0.57)$ & $8(1.15)$ & .39 & $1(0.13)$ & $7(0.91)$ & .07 \\
\hline Rheumatoid arthritis & $1(0.33)$ & $4(1.31)$ & .72 & $13(1.87)$ & $11(1.58)$ & .38 & $16(2.08)$ & $6(0.78)$ & .02 \\
\hline Coagulopathy & $4(1.31)$ & $3(0.98)$ & 1.00 & $14(2.01)$ & 27 (3.87) & .13 & $7(0.91)$ & $18(2.34)$ & .05 \\
\hline Obesity & $2(0.66)$ & $12(3.93)$ & .90 & $22(3.16)$ & $38(5.45)$ & .15 & $33(4.29)$ & $27(3.51)$ & .24 \\
\hline Weight loss & $8(2.62)$ & 0 & - & $6(0.86)$ & $5(0.72)$ & .54 & $10(1.30)$ & $10(1.30)$ & .84 \\
\hline Fluid and electrolyte disorder & $20(6.56)$ & 14 (4.59) & .27 & $91(13.06)$ & $69(9.90)$ & .001 & $94(12.22)$ & $70(9.10)$ & .005 \\
\hline Blood loss anemia & $1(0.33)$ & $2(0.66)$ & 1.00 & $2(0.29)$ & $1(0.14)$ & .59 & $5(0.65)$ & $1(0.13)$ & .11 \\
\hline Deficiency anemia & $1(0.33)$ & $3(0.98)$ & .64 & $8(1.15)$ & $9(1.29)$ & .89 & $18(2.34)$ & $8(1.04)$ & .03 \\
\hline Alcohol abuse & 0 & $2(0.66)$ & .51 & $2(0.29)$ & $8(1.15)$ & .12 & $5(0.65)$ & $11(1.43)$ & .18 \\
\hline Drug abuse & $1(0.33)$ & $1(0.33)$ & 1.00 & $1(0.14)$ & $1(0.14)$ & 1.00 & $3(0.39)$ & $3(0.39)$ & 1.00 \\
\hline Psychoses & 0 & $2(0.66)$ & .51 & $3(0.43)$ & $1(0.14)$ & .33 & $8(1.04)$ & $3(0.39)$ & .10 \\
\hline Depression & $8(2.62)$ & $18(5.90)$ & .25 & $35(5.02)$ & 25 (3.59) & .04 & $47(6.11)$ & $33(4.29)$ & .04 \\
\hline
\end{tabular}

Note. ${ }^{*}$ Probability of estimates from tests of association $\left(\left(^{2}\right)\right.$ or variance $\left(\mathrm{T}^{2}\right)$ between characteristics and sex

Most observations among three discrete ER-based patient women having diagnosis vs. 50\% men having a diagnosis. categories were made in ER history category. The distri- When it came to race categories, African American women bution of men and women were varied across all patient categories (scheduled: $7.11 \%$ women and $10.11 \%$ men; true emergencies: $17.90 \%$ women and $21.46 \%$ men; ER history: $20.72 \%$ women and $22.70 \%$ men) with men having more observations than women across all categories. There were more observations on heart block than any other diagnoses which were used as inclusion criterion. The most difference in terms of heart block diagnosis between men and women was observed in the scheduled category, with $31 \%$ and Hispanic women had more observations than their male counterparts in true emergency and ER history categories. Primary expected payer distribution amongst the genders was even across all patient categories. There were slight differences in certain intersections of payer and patient category, i.e., $51 \%$ scheduled male patients had Medicare as primary payer Medicare whereas $38 \%$ of women in the same category had Medicare as a primary payer. Most patients had 3 or more comorbidities across all categories irrespective of 
gender. Of note was the difference observed in scheduled patients with $27.05 \%$ women having 3 or more comorbidities and $49 \%$ of men having 3 or more comorbidities. In observations of succinct comorbidities, the maximum difference was observed in hypothyroidism (13.11\% for women and 5.90\% in men in the scheduled, and $10.76 \%$ in women and $5.60 \%$ in women in the true emergency categories respectively with $p \leq .0001)$, fluid and electrolyte disorders (13.06\% in women and $9.09 \%$ in men with $p=.0001$ ). Risk for female pacemaker across all categories and all categories combined were not statistically significant. Of note was Hispanic race (OR: 1.518; 95\% CI: 1.049-2.196), and certain comorbidities like peri-vascular disorder (OR: 1.657; 95\% CI: 1.182-2.322) and hypothyroidism (OR 0.381; 95\% CI: 0.161-0.505) had an effect on the odds for female pacemaker (see Table 2).

Table 3. Stochastic model: Binomial logit regression predicting effect of characteristics on patient category and female pacemaker

\begin{tabular}{|c|c|c|c|c|c|c|c|c|c|c|c|c|}
\hline \multirow[b]{2}{*}{ Effect } & \multicolumn{3}{|c|}{ ER History } & \multicolumn{3}{|c|}{ True Emergencies } & \multicolumn{3}{|c|}{ Scheduled } & \multicolumn{3}{|c|}{ Female pacemaker } \\
\hline & OR & $\begin{array}{l}95 \% \text { CI } \\
\text { lower }\end{array}$ & $\begin{array}{l}95 \% \\
\text { CI } \\
\text { upper }\end{array}$ & OR & $\begin{array}{l}95 \% \\
\text { CI } \\
\text { lower }\end{array}$ & $\begin{array}{l}95 \% \\
\text { CI } \\
\text { upper }\end{array}$ & OR & $\begin{array}{l}95 \% \text { CI } \\
\text { lower }\end{array}$ & $\begin{array}{ll}95 \% & \text { CI } \\
\text { upper } & \end{array}$ & OR & $\begin{array}{l}95 \% \mathrm{CI} \\
\text { lower }\end{array}$ & $\begin{array}{ll}95 \% & \text { CI } \\
\text { upper } & \end{array}$ \\
\hline $\begin{array}{l}\text { Patient Category (ref: } \\
\text { true emergencies) } \\
\text { ER history } \\
\text { Scheduled }\end{array}$ & NA & - & - & NA & - & - & NA & - & - & $\begin{array}{l}0.908 \\
1.113\end{array}$ & $\begin{array}{l}0.722 \\
0.820\end{array}$ & $\begin{array}{l}1.141 \\
1.511\end{array}$ \\
\hline Female & 1.136 & 0.921 & 1.402 & 0.954 & 0.771 & 1.180 & 0.857 & 0.647 & 1.137 & NA & - & - \\
\hline \multicolumn{13}{|l|}{ Race (ref=Hispanic) } \\
\hline White & 1.190 & 0.831 & 1.703 & 1.029 & 0.717 & 1.475 & 0.669 & 0.388 & 1.154 & 1.518 & 1.049 & 2.196 \\
\hline Black & 1.197 & 0.744 & 1.926 & 0.711 & 0.443 & 1.142 & 1.549 & 0.686 & 3.499 & 0.858 & 0.527 & 1.396 \\
\hline Age & 0.987 & 0.975 & 0.999 & 1.000 & 0.988 & 1.013 & 1.022 & 1.006 & 1.039 & 0.986 & 0.974 & 0.998 \\
\hline Length of Stay & 0.972 & 0.944 & 0.998 & 1.007 & 0.979 & 1.035 & 1.070 & 1.015 & 1.127 & 0.968 & 0.940 & 0.996 \\
\hline \multicolumn{13}{|l|}{$\begin{array}{l}\text { Payer (ref: private } \\
\text { insurance) }\end{array}$} \\
\hline Medicare & 0.932 & 0.617 & 1.408 & 1.279 & 0.852 & 1.920 & 0.722 & 0.424 & 1.231 & 1.103 & 0.725 & 1.678 \\
\hline Medicaid & 0.345 & 0.164 & 0.735 & 2.281 & 1.058 & 4.917 & 2.287 & 0.599 & 8.731 & 0.634 & 0.296 & 1.360 \\
\hline Brady & 0.977 & 0.757 & 1.262 & 0.959 & 0.743 & 1.236 & 1.124 & 1.006 & 1.039 & 0.867 & 0.667 & 1.125 \\
\hline Block & 1.480 & 1.055 & 2.077 & 0.594 & 0.417 & 0.847 & 1.192 & 0.755 & 1.882 & 1.163 & 0.821 & 1.649 \\
\hline \multicolumn{13}{|l|}{ Comorbidities (ref $\geq 3$ ) } \\
\hline No comorbidity & 0.689 & 0.289 & 1.642 & 1.778 & 0.669 & 4.727 & 0.767 & 0.267 & 2.205 & 0.873 & 0.353 & 2.159 \\
\hline 1 comorbidity & 0.526 & 0.302 & 0.918 & 1.354 & 0.754 & 2.432 & 2.026 & 0.880 & 4.666 & 1.296 & 0.727 & 2.311 \\
\hline 2 comorbidities & 1.335 & 0.861 & 2.071 & 1.099 & 0.716 & 1.688 & 0.621 & 0.383 & 1.007 & 1.644 & 1.047 & 2.582 \\
\hline Congestive Heart & 1.106 & 0.852 & 1.435 & 0.853 & 0.655 & 1.109 & 1.112 & 0.765 & 1.617 & 0.723 & 0.552 & 0.947 \\
\hline Cardiac Arrhythmias & 0.664 & 0.535 & 0.825 & 1.539 & 1.230 & 1.925 & 0.966 & 0.723 & 1.292 & 1.173 & 0.935 & 1.472 \\
\hline Valvular disease & 1.154 & 0.882 & 1.512 & 1.113 & 0.846 & 1.463 & 0.655 & 0.468 & 0.918 & 1.006 & 0.762 & 1.328 \\
\hline Pul. Circ. disorder & 1.024 & 0.636 & 1.651 & 1.499 & 0.899 & 2.499 & 0.476 & 0.254 & 0.892 & 0.596 & 0.361 & 0.984 \\
\hline Peri- vascular & 1.154 & 0.893 & 1.492 & 1.081 & 0.782 & 1.494 & 0.689 & 0.460 & 1.031 & 1.657 & 1.182 & 2.322 \\
\hline Hypertension U & 1.154 & 0.838 & 1.492 & 0.927 & 0.714 & 1.203 & 0.868 & 0.617 & 1.221 & 0.789 & 0.606 & 1.028 \\
\hline Hypertension C & 1.078 & 0.591 & 1.966 & 0.887 & 0.480 & 1.640 & 0.996 & 0.440 & 2.251 & 0.971 & 0.522 & 1.808 \\
\hline Paralysis & 6.385 & 0.952 & 42.799 & 0.138 & 0.023 & 0.827 & $>999.999$ & $<0.001$ & $>999.999$ & 1.399 & 0.273 & 7.163 \\
\hline Other neurological & 0.685 & 0.453 & 1.037 & 1.170 & 0.765 & 1.791 & 1.600 & 0.823 & 3.111 & 1.595 & 1.031 & 2.470 \\
\hline Chronic pulmonary & 0.731 & 0.558 & 0.959 & 1.048 & 0.794 & 1.382 & 1.669 & 1.105 & 2.520 & 1.155 & 0.871 & 1.532 \\
\hline Diabetes UC & 0.943 & 0.745 & 1.193 & 1.026 & 0.810 & 1.300 & 1.096 & 0.800 & 1.502 & 1.187 & 0.932 & 1.512 \\
\hline Diabetes C & 0.721 & 0.450 & 1.157 & 1.227 & 0.755 & 1.993 & 1.305 & 0.643 & 2.646 & 1.240 & 0.757 & 2.033 \\
\hline Hypothyroidism & 1.169 & 0.889 & 1.537 & 1.029 & 0.781 & 1.357 & 0.701 & 0.494 & 0.997 & 0.381 & 0.287 & 0.505 \\
\hline Renal failure & 0.973 & 0.533 & 1.775 & 1.053 & 0.569 & 1.948 & 0.996 & 0.441 & 2.250 & 1.573 & 0.844 & 2.932 \\
\hline Liver disease & 1.637 & 0.734 & 3.650 & 0.606 & 0.282 & 1.302 & 1.005 & 0.319 & 3.160 & 0.383 & 0.161 & 0.914 \\
\hline Peptic ulcer w/o B & 1.190 & 0.369 & 3.840 & 0.625 & 0.188 & 2.076 & 1.851 & 0.217 & 15.824 & 2.982 & 0.841 & 10.581 \\
\hline HIV/AIDS & 3.838 & 0.182 & 80.817 & 0.249 & 0.011 & 5.568 & $>999.999$ & $<0.001$ & $>999.999$ & $>999.999$ & $<0.001$ & $>999.999$ \\
\hline Metastatic cancer & $>999.9$ & $<0.001$ & $>999.9$ & 0.598 & 0.089 & 4.037 & 0.173 & 0.022 & 1.333 & 2.372 & 0.199 & 28.248 \\
\hline Tumor w/o M & 1.291 & 0.499 & 3.342 & 0.700 & 0.288 & 1.706 & 1.061 & 0.319 & 3.534 & 2.334 & 0.827 & 6.587 \\
\hline Rheumatoid arthritis & 1.145 & 0.635 & 2.065 & 0.774 & 0.432 & 1.386 & 1.207 & 0.522 & 2.791 & 0.560 & 0.301 & 1.043 \\
\hline Coagulopathy & 1.576 & 0.924 & 2.687 & 0.473 & 0.284 & 0.789 & 2.250 & 0.864 & 5.860 & 2.298 & 1.290 & 4.094 \\
\hline Obesity & 1.053 & 0.711 & 1.559 & 0.906 & 0.611 & 1.342 & 1.103 & 0.637 & 1.913 & 0.989 & 0.660 & 1.482 \\
\hline Weight loss & 0.573 & 0.252 & 1.304 & 1.161 & 0.506 & 2.664 & $>999.999$ & $<0.001$ & $>999.999$ & 1.766 & 0.754 & 4.135 \\
\hline Fluid and electrolyte & 1.092 & 0.836 & 1.426 & 0.716 & 0.548 & 0.935 & 1.688 & 1.110 & 2.567 & 0.572 & 0.434 & 0.755 \\
\hline Blood loss anemia & 0.997 & 0.293 & 3.388 & 2.218 & 0.568 & 8.668 & 0.279 & 0.065 & 1.202 & 0.482 & 0.131 & 1.769 \\
\hline Deficiency anemia & 0.775 & 0.414 & 1.451 & 1.111 & 0.586 & 2.107 & 1.530 & 0.522 & 4.484 & 0.754 & 0.392 & 1.452 \\
\hline Alcohol abuse & 0.525 & 0.230 & 1.202 & 1.516 & 0.649 & 3.541 & 1.669 & 0.366 & 7.612 & 3.546 & 1.353 & 9.292 \\
\hline Drug abuse & 0.632 & 0.155 & 2.580 & 3.115 & 0.559 & 17.353 & 0.542 & 0.089 & 3.296 & 0.448 & 0.112 & 1.793 \\
\hline Psychoses & 0.696 & 0.239 & 2.030 & 1.947 & 0.596 & 6.359 & 0.584 & 0.116 & 2.949 & 0.563 & 0.191 & 1.665 \\
\hline Depression & 0.904 & 0.640 & 1.276 & 1.124 & 0.788 & 1.603 & 0.977 & 0.610 & 1.563 & 0.660 & 0.462 & 0.942 \\
\hline
\end{tabular}


Medicare beneficiaries with primary pacemaker were almost $65 \%$ less likely to be in the ER history category when compared with those having private insurance (OR: $0.35 ; 95 \%$ CI: 0.16-0.74). However, they had a 2-fold greater likelihood for being true emergencies than patients with private insurance (OR: 2.28; 95\% CI: 1.06-4.91). Patients having heart block as an inpatient discharge diagnosis were $48 \%$ more likely to be in the ER history category when compared with those who did not have heart block alone as a discharge diagnosis (95\%CI: 1.06-2.08). Conversely, patients with heart block alone (not including a diagnosis of bradycardia) had only half the likelihood to be in the true emergency category when compared with those who did not have it (OR: 0.594; 95\% CI: 0.42- 0.85). Cardiac arrhythmias decreased likelihood for patients to be in the ER history category by $44 \%$ (OR: 0.66; 95\% CI: 0.54-0.83), although it increased likelihood of patients to be in the true emergency category by $54 \%$ (OR: 1.54; 95\% CI: 1.24-1.93). Any diagnosis for coagulopathy, on the other hand, decreased the likelihood for patients to be true emergencies by more than 50\% (OR: $0.47 ; 95 \% \mathrm{CI}$ : 0.28-0.79). Patient demographic characteristics did not have significant effect on their likelihood of being scheduled admissions. However, not having comorbidities like pulmonary circulation disorder (OR: 0.48; 95\% CI: 0.25-0.89) and having comorbidities like chronic pulmonary disease and fluid and electrolyte disorders had a 67\% (95\% CI: 1.11- 2.52) and 69\% (95\% CI: 1.11-2.57) increased likelihood, respectively, for patients to be in the scheduled category (see Table 3).

Likelihood for women to receive pacemakers increased by $52 \%$ in white patients when compared with those who were from other races, not including black (OR: 1.52; 95\% CI: 1.05-2.19). Likelihood for women to receive pacemakers also increased by $64 \%$ in patients having 2 comorbidities in their discharge record when compared with those who had 3 or more comorbidities (OR: 1.64; 95\% CI: 1.05-2.58). Female patients with peri-vascular disease were $66 \%$ more likely (OR: 1.66; 95\% CI: 1.18-2.32), those with neurological disorders were $60 \%$ more likely (OR: 1.6; 95\% CI: 1.03-2.47), those with coagulopathy were 2 and half times more likely (OR: 2.29; 95\% CI: 1.29-4.09) and those with fluid and electrolyte disorders were $43 \%$ less likely (OR: 0.57 ; $95 \%$ CI: 0.43-0.76) have a pacemaker, when compared with males with the same diagnoses (see Table 3).

We could secure decision tree algorithms for predicting ER history, True Emergency and Female pacemakers. According to our non-linear modeling, patients with cardiac arrhythmias, not having deficiency anemia, not having chronic pulmonary disease, having a length of inpatient stay more than or equal to 6.5 days, not being black, not having heart block as a discrete inpatient discharge diagnosis and of age more than 76.5 years had the highest chance of having ER history (see Table 4). Patients with cardiac arrhythmias, with fluid and electrolyte disorders, experiencing a length of inpatient stay less than 4.5 days and having depression and coagulopathy had the highest chance of being true emergencies. Patients receiving a permanent initial pacemaker without fluid and electrolyte disorder, renal failure, of more than 83.5 years of age, experiencing a length of inpatient stay more than 3.5 days and not having pulmonary circulation disorder or deficiency anemia were more likely to be female (see Table 4).

\section{Discussion}

Consistent with previous literature, we did not find any significant differences in terms of sex as an effect on primary pacemaker implants as well as ER use previous to implant. However subtle differences were observed in discrete patient characteristics like comorbidities, race and primary expected payer in sex-based and ER-utilization based stratification of subgroups. Of note was the importance of hypothyroidism as a point of difference between men and women receiving primary pacemaker. The thyroid hormone affects the heart and the vascular system at many levels and hypothyroidism is symptomatic by bradycardia, ${ }^{[28]}$ which was a primary inclusion criterion in our study. Hypothyroidism is more clinically manifest in older populations, and more so in older women when compared to men of comparable age groups ${ }^{[29]}$ With a mean age of 79 years for female and 77 years in male patients in our sample, this observation was expected. The intersection of race white and primary payer Medicare as a dominant subgroup in sex stratified characterization of patients was also expected because of the age distribution of patients. Most patients were in the ER history category and there were no tangible differences in sex stratification of observations. This can be explained by the fact that inpatient, especially surgical procedures, are increasingly becoming associated with ER visits and there are no apparent sex disparities in ER use in the US. In our study, there were more patients in the ER history category than true emergency category (which would have been an expected observation) because our sample of patients were relatively healthy compared to other patients with cardiac rhythm management needs. This was due to the fact that our selection criterion only retained patients with bradycardia and/or heart block in need of initial pacemaker as a primary procedure; characteristics which are less critical than atrio-ventricular fibrillation requiring implantable cardioverter defibrillators, for example. Outputs of our predictive models using stochastic methodology were very different from the outputs of our predictive models using recursive partitioning method, except for certain predictions 
around comorbidities. Since our non-linear models exposed the data to a more detailed and granular segmentation when compared to our linear explorations, we can confidently say that these overlaps in observations, e.g., absence of fluid and electrolyte disorders predicting female pacemaker, can be deduced as being really important markers for sex-specific differences in risk for primary pacemakers. We could also deduce that white implantees with heart block and longer inpatient stays were more likely to have visited the ER before they receive a transplant. This finding indicates that this specific population may be very sick, as evidenced by the quantity of health resource utilization, and needs special attention from the health care system. Fluid and electrolyte disorders were a consistent attribute in both types of models in predicting true emergency patients. This finding is perhaps pertinent for clinical definitions for emergency surgeries (scheduled on the same day patient has the first symptomrelated encounter with the healthcare system) and for surveillance of population health for cardiac insufficiencies and rhythm management.

Table 4. Recursive partitioning models: Relative importance of variables for predicting pacemaker outcomes

\begin{tabular}{|c|c|c|c|c|}
\hline Variable & Relative Importance & Level Value & Predictive Probability & Decision \\
\hline \multicolumn{5}{|l|}{ ER History } \\
\hline Cardiac arrhythmias & 1.00 & $\begin{array}{l}1 \\
0\end{array}$ & $\begin{array}{l}0.70 \\
0.43\end{array}$ & $\begin{array}{l}\text { Yes } \\
\text { No }\end{array}$ \\
\hline Length of Stay & 0.8973489615 & $\begin{array}{l}<6.5 \text { days } \\
\geq 6.5 \text { days }\end{array}$ & $\begin{array}{l}0.40 \\
0.91\end{array}$ & $\begin{array}{l}\text { No } \\
\text { Yes }\end{array}$ \\
\hline Heart Block & 0.8886979344 & $\begin{array}{l}1 \\
0\end{array}$ & $\begin{array}{l}0.33 \\
0.81\end{array}$ & $\begin{array}{l}\text { No } \\
\text { Yes }\end{array}$ \\
\hline Race Black & 0.7714698832 & $\begin{array}{l}1 \\
0\end{array}$ & $\begin{array}{l}0.48 \\
0.53\end{array}$ & $\begin{array}{l}\text { No } \\
\text { Yes }\end{array}$ \\
\hline Race Hispanic & 0.7520614393 & $\begin{array}{l}1 \\
0\end{array}$ & $\begin{array}{l}0.36 \\
0.37\end{array}$ & $\begin{array}{l}\text { No } \\
\text { No }\end{array}$ \\
\hline Age & 0.7381504145 & $\begin{array}{l}<76.5 \text { years } \\
\geq 76.5 \text { years }\end{array}$ & $\begin{array}{l}0.50 \\
0.59\end{array}$ & $\begin{array}{l}\text { No } \\
\text { Yes }\end{array}$ \\
\hline Chronic Pulmonary Disease & 0.6343981574 & $\begin{array}{l}1 \\
0\end{array}$ & $\begin{array}{l}0.48 \\
0.53\end{array}$ & $\begin{array}{l}\text { No } \\
\text { Yes }\end{array}$ \\
\hline Deficiency Anemia & 0.6343981574 & $\begin{array}{l}1 \\
0\end{array}$ & $\begin{array}{l}0.65 \\
0.49\end{array}$ & $\begin{array}{l}\text { Yes } \\
\text { No }\end{array}$ \\
\hline Other neurological disorders & 0.6101971359 & - & - & - \\
\hline \multicolumn{5}{|l|}{ True ER } \\
\hline Cardiac arrhythmias & 1 & $\begin{array}{l}1 \\
0\end{array}$ & $\begin{array}{l}0.68 \\
0.39\end{array}$ & $\begin{array}{l}\text { Yes } \\
\text { No }\end{array}$ \\
\hline Length of stay & 0.821827677 & $\begin{array}{l}<4.5 \text { days } \\
\geq 4.5\end{array}$ & $\begin{array}{l}0.63 \\
0.41\end{array}$ & $\begin{array}{l}\text { Yes } \\
\text { No }\end{array}$ \\
\hline Coagulopathy & 0.7343045513 & $\begin{array}{l}1 \\
0\end{array}$ & $\begin{array}{l}0.36 \\
0.68\end{array}$ & $\begin{array}{l}\text { No } \\
\text { Yes }\end{array}$ \\
\hline Heart block & 0.7128874801 & $\begin{array}{l}1 \\
0\end{array}$ & $\begin{array}{l}0.33 \\
0.19\end{array}$ & $\begin{array}{l}\text { No } \\
\text { No }\end{array}$ \\
\hline Hypertension unspecified & 0.664613768 & $\begin{array}{l}1 \\
0\end{array}$ & $\begin{array}{l}0.41 \\
0.11\end{array}$ & $\begin{array}{l}\text { No } \\
\text { No }\end{array}$ \\
\hline Fluid and Electrolyte disorder & 0.6493967415 & $\begin{array}{l}1 \\
0\end{array}$ & $\begin{array}{l}0.51 \\
0.41\end{array}$ & $\begin{array}{l}\text { Yes } \\
\text { No }\end{array}$ \\
\hline Depression & 0.4389390271 & $\begin{array}{l}1 \\
0\end{array}$ & $\begin{array}{l}0.75 \\
0.22\end{array}$ & $\begin{array}{l}\text { Yes } \\
\text { No }\end{array}$ \\
\hline \multicolumn{5}{|l|}{ Female pacemaker } \\
\hline Hypothyroidism & 1 & $\begin{array}{l}1 \\
0\end{array}$ & $\begin{array}{l}0.38 \\
0.46\end{array}$ & $\begin{array}{l}\text { No } \\
\text { No }\end{array}$ \\
\hline Fluid and Electrolyte disorder & 0.5433085621 & $\begin{array}{l}1 \\
0\end{array}$ & $\begin{array}{l}0.42 \\
0.66\end{array}$ & $\begin{array}{l}\text { No } \\
\text { Yes }\end{array}$ \\
\hline Age & 0.5152513279 & $\begin{array}{l}<83.5 \text { years } \\
\geq 83.5\end{array}$ & $\begin{array}{l}0.49 \\
0.79\end{array}$ & $\begin{array}{l}\text { No } \\
\text { Yes }\end{array}$ \\
\hline Length of stay & 0.4942096927 & $\begin{array}{l}<3.5 \text { days } \\
\geq 3.5 \text { days }\end{array}$ & $\begin{array}{l}0.31 \\
0.78\end{array}$ & $\begin{array}{l}\text { No } \\
\text { Yes }\end{array}$ \\
\hline Pulmonary circulation disorder & 0.4366073401 & $\begin{array}{l}1 \\
0\end{array}$ & $\begin{array}{l}0.41 \\
0.61\end{array}$ & $\begin{array}{l}\text { No } \\
\text { Yes }\end{array}$ \\
\hline Renal Failure & 0.4038033386 & $\begin{array}{l}1 \\
0\end{array}$ & $\begin{array}{l}0.53 \\
0.38\end{array}$ & $\begin{array}{l}\text { Yes } \\
\text { No }\end{array}$ \\
\hline Deficiency anemia & 0.3268266559 & $\begin{array}{l}1 \\
0 \\
\end{array}$ & $\begin{array}{l}0.41 \\
0.60 \\
\end{array}$ & $\begin{array}{l}\text { No } \\
\text { Yes }\end{array}$ \\
\hline
\end{tabular}

Note. There were no variables predictive of scheduled pacemaker implants 


\section{Limitations}

Unfortunately, our analysis lacked the data strength to explore more meaningful overlaps. Due to the same limitation, we could not generate a non-linear predictive model for scheduled patients. A significant limitation of our study was our inability to compare true emergencies and ER history patients in the ER setting since true emergencies were selected from the SID and not the SEDD by virtue of appointed definitions, although both groups have had ER use previous to implant.

\section{Conclusions}

A novel outcome of this study pertains to our schema of categorizing patients around their ER utilization prior to surgery. We did not depend on a single criterion (e.g., utilization flag) in one healthcare setting but used data from two different settings (inpatient and ER) to form our discrete categories (scheduled, ER history and True Emergencies), which possibly made our outcome measures around previous ER use more valid and robust than would have been the case otherwise. Additionally, this is the first time to our knowledge that different HCUP products (the SEDD and the SID) were used together to follow patients between different settings and years. This was made possible because HCUP Florida products keep the patient identifier unique to patients across settings and time. Our method of data compilation opens up possibilities to study numerous hospital based events at the patient level across time using publicly available hospital discharge databases. Another novel methodological aspect of our study pertains to exploring interactions between gender and ER utilization around pacemaker implant, a cardiac event. As underlined earlier, cardiac events display high gender disparity and have high association with ER use. We did not find any previous study exploring these interactions. Future investigations in this subject should involve a larger sample size in order to carry out non-linear models of exploration along with stochastic analyses in order to increase validity of findings.

\section{REFERENCES}

[1] Brunner M, Manfred O, Annette G, et al. Long-term survival after pacemaker implantation Prognostic importance of gender and baseline patient characteristics. European Heart Journal. 2004; 25(1): 88-95. PMid: 14683747. http://dx.doi.org/10.1016/j.ehj .2003 .10 .022

[2] Kurtz SM, Ochoa JA, Edmund LMS, et al. Implantation trends and patient profiles for pacemakers and implantable cardioverter defibrillators in the United States: 1993-2006. Pacing and Clinical Electrophysiology. 2010; 33(6): 705-711. PMid: 20059714. http://dx.doi.org/10.1111/j.1540-8159.2009.02670.x

[3] Jung W, Rillig A, Birkemeyer R, et al. Advances in remote monitoring of implantable pacemakers, cardioverter defibrillators and cardiac resynchronization therapy systems. Journal of Interventional Cardiac Electrophysiology. 2008; 23(1): 73-85. PMid: 18821006. http://dx.doi.org/10.1007/s10840-008-9311-5

[4] Silverman BG, Gross TP, Kaczmarek RG, et al. The epidemiology of pacemaker implantation in the United States. Public Health Reports. 1995; 110(1): 42. PMid: 7838942.

[5] Brown DW, Croft JB, Giles WH, et al. Epidemiology of pacemaker procedures among Medicare enrollees in 1990, 1995, and 2000. The American Journal of Cardiology. 2005; 95(3): 409-411. PMid: 15670557. http://dx.doi.org/10.1016/j.amjcard. 2 004.09 .046

[6] Gregoratos G, Abrams J, Epstein AE, et al. ACC/AHA/NASPE 2002 guideline update for implantation of cardiac pacemakers and antiarrhythmia devices—summary article: a report of the American College of Cardiology/American Heart Association Task Force on Practice Guidelines (ACC/AHA/NASPE Committee to Update the 1998 Pacemaker Guidelines). Journal of the American College of Cardiology. 2002; 40(9): 1703-1719. http://dx.doi.org/10.10 16/S0735-1097 (02) 02528-7

[7] Epstein AE, Dimarco JP, Ellenbogen KA, et al. ACC/AHA/HRS 2008 guidelines for device-based therapy of cardiac rhythm abnor- malities: a report of the American College of Cardiology/American Heart Association Task Force on Practice Guidelines (Writing Committee to Revise the ACC/AHA/NASPE 2002 Guideline Update for Implantation of Cardiac Pacemakers and Antiarrhythmia Devices) developed in collaboration with the American Association for Thoracic Surgery and Society of Thoracic Surgeons. Journal of the American College of Cardiology. 2008; 51(21): e1-e62. PMid: 18498951 http://dx.doi.org/10.1016/j.jacc.2008.02.032

[8] Hasan J, Derek C, Mariuca VN, et al. Predictors for permanent pacemaker requirement after transcatheter aortic valve implantation with the CoreValve bioprosthesis. American Heart Journal. 2009; 157(5): 860-866. PMid: 19376312. http://dx.doi.org/10.1016/j.a hj. 2009.02.016

[9] Raviele A, Giada F, Menozzi C, et al. A randomized, double-blind, placebo-controlled study of permanent cardiac pacing for the treatment of recurrent tilt-induced vasovagal syncope. The vasovagal syncope and pacing trial (SYNPACE). European Heart Journal. 2004; 25(19): 1741-1748. PMid: 15451153. http://dx.doi.org/10. 1016/j.ehj.2004.06.031

[10] Brignole M, Auricchio A, Baronesquivias G, et al. 2013 ESC Guidelines on cardiac pacing and cardiac resynchronization therapy The Task Force on cardiac pacing and resynchronization therapy of the European Society of Cardiology (ESC). Developed in collaboration with the European Heart Rhythm Association (EHRA). European Heart Journal. 2013; eht150.

[11] Brignole M, Ungar A, Casagranda I, et al. Prospective multicentre systematic guideline-based management of patients referred to the Syncope Units of general hospitals. Europace. 2010; 12: 109-118.

[12] Herlitz J, Lindqvist J. Improvement of ED prediction of cardiac mortality among patients with symptoms suggestive of acute myocardial infarction. The American Journal of Emergency Medicine. 1997; 15(1): 1-7. http://dx.doi.org/10.1016/S0735-6757(97)9 0038-3 
[13] Alan M, Hollander JE, David G, et al. Primary results of the Rapid Emergency Department Heart Failure Outpatient Trial (REDHOT): A multicenter study of B-type natriuretic peptide levels, emergency department decision making, and outcomes in patients presenting with shortness of breath. Journal of the American College of Cardiology. 2004; 44(6): 1328-1333. PMid: 15364340. http://dx.doi.org/10.1016/j.jacc. 2004.06.015

[14] Pitts SR, Niska RW, Xu J, et al. National Hospital Ambulatory Medical Care Survey: 2006 emergency department summary. National health statistics reports; no 7. 2008. Hyattsville, National Center for Health Statistics. 2008; 7(7): 1-38.

[15] Ning T, John S, Hsia RY, et al. Trends and characteristics of US emergency department visits, 1997-2007. JAMA. 2010; 304(6): 664-670. PMid: 20699458. http://dx.doi.org/10.1001/jama.2010.1 112

[16] Schuur JD, Venkatesh AK. The growing role of emergency departments in hospital admissions. New England Journal of Medicine; 2012; 367(5): 391-393. PMid: 22784039. http://dx.doi.org/1 $0.1056 /$ NEJMp1204431

[17] Pitts SR, Carrier ERRich EC, Kellermann AL. Where Americans get acute care: increasingly, it's not at their doctor's office. Health Affairs. 2010; 29(9): 1620-1629. PMid: 20820017. http://dx.d oi.org/10.1377/hlthaff.2009.1026

[18] Bird CE, Shugarman LR, Lynn J. Age and gender differences in health care utilization and spending for Medicare beneficiaries in their last years of life. Journal of Palliative Medicine. 2002; 5(5): 705-712. PMid: 12572969. http://dx.doi.org/10.1089/109 662102320880525

[19] Keene J, Li X. Age and gender differences in health service utilization. Journal of Public Health. 2005; 27(1): 74-79. PMid: 15637107 http://dx.doi.org/10.1093/pubmed/fdh208

[20] DeVon HA, Zerwic JJ. Symptoms of acute coronary syndromes: are there gender differences? A review of the literature. Heart \& Lung: The Journal of Acute and Critical Care. 2002; 31(4): 235-245. http://dx.doi.org/10.1067/mhl.2002.126105

[21] Blomkalns AL, Chen AY, JS Hochman, et al. Gender disparities in the diagnosis and treatment of non-ST-segment elevation acute coronary syndromes: large-scale observations from the CRUSADE
(Can Rapid Risk Stratification of Unstable Angina Patients Suppress Adverse Outcomes With Early Implementation of the American College of Cardiology/American Heart Association Guidelines) national quality improvement initiative. Journal of the American College of Cardiology. 2005; 45(6): 832-837. PMid: 15766815. http://dx.doi.org/10.1016/j.jacc.2004.11.055

[22] Milner KA, Funk M, Richards S, et al. Gender differences in symptom presentation associated with coronary heart disease. The American Journal of Cardiology. 1999; 84(4): 396-399. http: //dx.doi.org/10.1016/S0002-9149(99)00322-7

[23] Shaw LJ, Miller DD, Romeis JC, et al. Gender differences in the noninvasive evaluation and management of patients with suspected coronary artery disease. Annals of Internal Medicine. 1994; 120(7): 559566. PMid: 8116993. http://dx.doi.org/10.7326/0003-481 9-120-7-199404010-00005

[24] Thomas T, Nancy H, Wayne R, et al. Heart disease and stroke statistics-2006 update a report from the American Heart Association Statistics Committee and Stroke Statistics Subcommittee. Circulation. 2006; 113(6): e85-e151. PMid: 16407573. http: //dx.doi.org/10.1161/CIRCULATIONAHA.105.171600

[25] Owens GM. Gender differences in health care expenditures, resource utilization, and quality of care. Journal of Managed Care Pharmacy. 2008; 14(3): S2.

[26] Correa-de-Araujo R, Stevens B, Moy E, et al. Gender differences across racial and ethnic groups in the quality of care for acute myocardial infarction and heart failure associated with comorbidities. Women's Health Issues. 2006; 16(2): 44-55. PMid: 16638521. http://dx.doi.org/10.1016/j.whi.2005.04.003

[27] David B, Kathryn W, Ann G, et al. Reasons for Escalating Pacemaker Implants. The American Journal of Cardiology. 2006; 98(1): 93-97. PMid: 16784928. http://dx.doi.org/10.1016/j.amjcard.2 006.01 .069

[28] Epstein FH, Klein I, Ojamaa K. Thyroid hormone and the cardiovascular system. New England Journal of Medicine. 2001; 344(7): 501-509. PMid: 11172193. http://dx.doi.org/10.1056/NEJ M200102153440707

[29] Morganti S, Ceda GP, Saccani M, et al. Thyroid disease in the elderly: sex-related differences in clinical expression. Journal of Endocrinological Investigation. 2004; 28 (11 Suppl Proceedings): 101-104. 